\title{
Surface loss probability of atomic hydrogen for different electrode cover materials investigated in $\mathrm{H}_{2}$-Ar low-pressure plasmas
}

\author{
M. Sode, ${ }^{1, \text { a) }}$ T. Schwarz-Selinger, ${ }^{1}$ W. Jacob, ${ }^{1}$ and H. Kersten ${ }^{2}$ \\ 1) Max-Planck-Institut für Plasmaphysik, Boltzmannstraße 2, D-85748 Garching, \\ Germany. \\ ${ }^{2)}$ Institute for Experimental and Applied Physics, Christian- \\ Albrechts-Universität zu Kiel, Leibnizstraße 11-19, D-24098 Kiel, \\ Germany.
}

(Dated: 2 July 2014)

In an inductively-coupled $\mathrm{H}_{2}$ - $\mathrm{Ar}$ plasma at a total pressure of $1.5 \mathrm{~Pa}$ the influence of the electrode cover material on selected line intensities of $\mathrm{H}, \mathrm{H}_{2}$, and $\mathrm{Ar}$ are determined by optical emission spectroscopy and actinometry for the electrode cover materials stainless steel, copper, tungsten, Macor ${ }^{\circledR}$, and aluminum. Hydrogen dissociation degrees for the considered conditions are determined experimentally from the measured emission intensity ratios. The surface loss probability $\beta_{\mathrm{H}}$ of atomic hydrogen is correlated with the measured line intensities and $\beta_{\mathrm{H}}$ values are determined for the considered materials. Without the knowledge of the atomic hydrogen temperature, $\beta_{\mathrm{H}}$ cannot be determined exactly. However, ratios of $\beta_{\mathrm{H}}$ values for different surface materials are in first order approximation independent of the atomic hydrogen temperature. Our results show that $\beta_{\mathrm{H}}$ of copper is equal to the value of stainless steel, $\beta_{\mathrm{H}}$ of Macor ${ }^{\circledR}$ and tungsten is about 2 times smaller and $\beta_{\mathrm{H}}$ of aluminum about 5 times smaller compared with stainless steel. The latter ratio is in reasonable agreement with literature. The influence of the atomic hydrogen temperature $T_{\mathrm{H}}$ on the absolute value is thoroughly discussed. For our assumption of $T_{\mathrm{H}}=600 \mathrm{~K}$ we determine a $\beta_{\mathrm{H}}$ for stainless steel of $0.39 \pm 0.13$.

PACS numbers: 52.25.-b, 52.27.Cm, 52.40.Hf, 52.70.Kz

Keywords: atomic hydrogen, surface loss probability, actinometry

a)Electronic mail: maik.sode@ipp.mpg.de 


\section{INTRODUCTION}

Plasma process technology plays an important role in a wide range of different industrial applications such as semiconductors, solar cells, and display manufacturing. Reactive plasmas are used for etching, coating and surface treatment. In a large variety of cases etching or deposition processes are based on the reactivity of the radical particles involved. Therefore, quantifying the radical particle densities is a first and important step for an improved understanding of plasma-surface-interaction processes.

In particular, $\mathrm{H}_{2}$-containing plasmas have a broad range of applications in surface engineering, for example in film deposition ${ }^{1-6}$, etching ${ }^{7}$ as well as hydrogenation, surface passivation, and oxide reduction ${ }^{8-11}$. Furthermore, $\mathrm{H}_{2}$-Ar mixtures were successfully applied for hydrogenation of thin film transistors ${ }^{12}$ and to control the surface properties of polymers ${ }^{13}$.

This study is focused on the radical species in a low temperature $\mathrm{H}_{2}$-Ar plasma, namely atomic hydrogen. To calculate the particle flux of atomic hydrogen onto the surrounding walls its concentration and temperature have to be known. The concentration is governed by production and loss processes. For low pressure plasmas atomic hydrogen is mainly produced by electron-induced dissociation of $\mathrm{H}_{2}$. This process is predominantly affected by the electron density and electron temperature of the respective plasma. In these plasmas the loss of atomic hydrogen is determined by the flux to the wall ${ }^{14-17}$. In our case, as in most other cases, the wall loss is determined by recombination of atomic hydrogen to form molecular hydrogen which desorbs from the surface. This wall loss is described by the surface loss probability $\beta_{\mathrm{H}} . \quad \beta_{\mathrm{H}}$ is a material specific property and is different for different materials. However, published values vary significantly between different experiments. In several publications the surface loss probability of atomic hydrogen for stainless steel or aluminum was determined in plasma experiments ${ }^{14,15,18-21}$. However, to our knowledge, values for other materials such as copper, Macor ${ }^{\circledR}$, and tungsten which are determined experimentally in the present work were not yet published. It is obvious that $\beta_{\mathrm{H}}$ is an important parameter for calculating the atomic hydrogen concentration. In a recent publication ${ }^{17}$ it was shown that for the correct description of the $\mathrm{H}$ atom density in a low-temperature plasma the knowledge of $\beta_{\mathrm{H}}$ is a mandatory prerequisite. Due to the strong correlation of the radical and ion densities ${ }^{17} \beta_{\mathrm{H}}$ also influences the density of the $\mathrm{H}$ ion species.

In this article we additionally discuss uncertainties inherent to the determination of $\beta_{\mathrm{H}}$. When comparing different literature results one recognizes that $\beta_{\mathrm{H}}$ values depend not only on the material but obviously also on the geometry of the plasma vessel and on the assumption about the $\mathrm{H}$ atom temperature. In this work, we compare $\beta_{\mathrm{H}}$ for different electrode materials for the identical setup and identical plasma parameters. It will be shown that the material of the electrode influences significantly the density of atomic hydrogen and, hence, the degree of $\mathrm{H}_{2}$ dissociation. The other plasma parameters (especially $n_{e}, T_{e}$, and $T_{g}$ ), however, are not affected. To change the material of the electrode exposed to the plasma, an electrode cover is used. The influence of the electrode cover material on the atomic hydrogen $(\mathrm{H})$ density as well as the $\mathrm{H}_{2}$ and Ar density is studied by optical emission spectroscopy (OES). The absolute atomic hydrogen density $n_{\mathrm{H}}$ is determined by actinometry. From the atomic hydrogen density the wall loss time and the wall loss probability are determined. 
In two preceding publications the applied plasma was characterized ${ }^{17,22}$ for quite similar plasma conditions as in the present case. The densities of the gas species, the radical species $\mathrm{H}$, the electrons and the ion species as well as the temperature of the gas and the electrons were obtained experimentally ${ }^{22}$. Furthermore, by a rate equation model the ion densities, the atomic hydrogen density and the electron temperature were studied theoretically ${ }^{17}$. In this study we complement these investigations by showing the influence of the material surrounding the plasma on the parameters, especially the surface loss probability of atomic hydrogen.

\section{EXPERIMENT}

\section{A. Experimental setup and Langmuir probe}

The experimental setup consists of a cylindrical stainless steel plasma chamber shown schematically in Fig. 1. A detailed description of the experimental set-up can be found in Ref. ${ }^{22}$. In short: The discharge is generated by inductive coupling using a planar coil with 5 turns and $100 \mathrm{~mm}$ in diameter. The coil is driven by a radio frequency $(r f)$ generator operating at $13.56 \mathrm{MHz}$ with a maximum power of 600 Watt (Dressler Cesar 136). The coil is separated from the vacuum chamber by a quartz dome ${ }^{23}$. The top part of the dome is $10 \mathrm{~mm}$ thick and acts as the dielectric window for the $r f$ power. The outer diameter $d_{e l}$ of the quartz dome and the stainless steel electrode which is located opposite to the dielectric window is $d_{e l}=2 r_{e l}=131 \mathrm{~mm}$ where $r_{e l}$ is the corresponding radius. The axial distance $l_{e l}$ between the electrode and the quartz window is $60 \mathrm{~mm}$. The plasma is mainly generated in the cylinder with the volume $V=\pi r_{e l}^{2} l_{e l}$ formed between electrode and window. At $r_{e l}$ there is no radial side wall between the electrode and dielectric window. The stainless steel vacuum chamber has a radius of $r_{c h}=125 \mathrm{~mm}$ and a total height of $l_{c h}=360 \mathrm{~mm}$.

To change the material of the electrode exposed to the plasma, a movable electrode cover is used. The studied electrode cover materials are stainless steel, copper, tungsten, Macor ${ }^{\circledR 24}$, and aluminum. The electrode cover is inserted directly under the upper electrode. With a load-lock system the transfer of the electrode cover occurs under vacuum so that a possible surface contamination of the main chamber is minimized. Inserting the cover reduces the axial distance $l_{e l}$ between the quartz window and the electrode cover by $2 \mathrm{~mm}$. The maximum transferable width of the electrode cover is $95 \mathrm{~mm}$. It therefore covers only $84 \%$ of the electrode. The shape of the electrode cover is a circular disk with $125 \mathrm{~mm}$ in diameter which is truncated at two opposite sides. It is schematically shown in Fig. 1. In order to reduce surface contamination on the electrode a $\mathrm{H}_{2}$-Ar plasma at $1.5 \mathrm{~Pa}$ with an Ar fraction of $f_{\mathrm{Ar}}=29 \%$ is operated for one hour before starting the measurements. The line of sight of the optical spectrometer runs along the long axis of the electrode cover (see also Fig. 1).

Measurements were conducted for a total gas pressure of $p=1.5 \mathrm{~Pa}$. The plasma chamber was pumped by a turbo molecular pump with Hohlweck stage to achieve good compression even for $\mathrm{H}_{2}$. The incoming gas flows were adjusted with mass-flow controllers. Mass spectrometer investigations have shown that the partial pressure ratios are not identical to the 


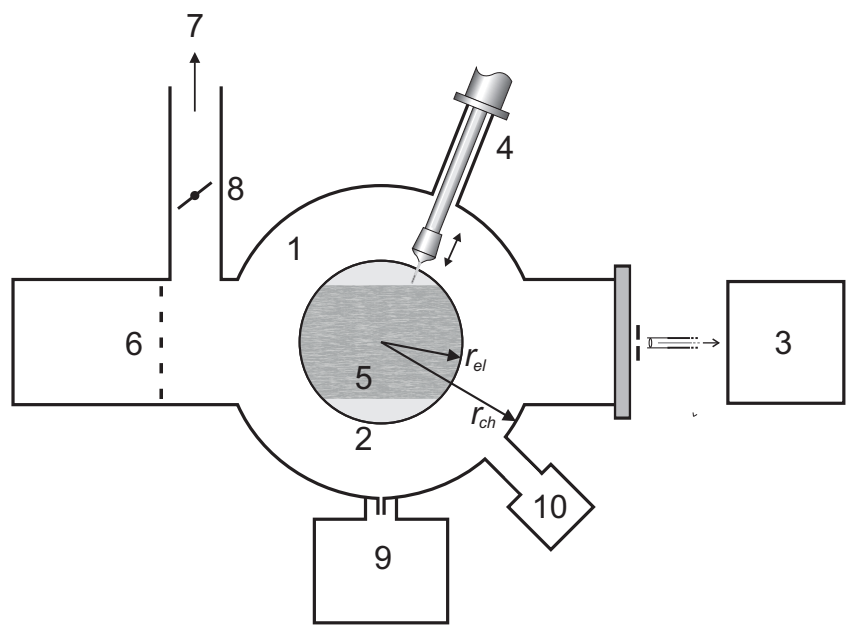

FIG. 1. Schematic representation of the plasma chamber (top view): (1) vacuum chamber made of stainless steel, (2) electrode, (3) optical emission spectroscopy, (4) Langmuir probe, (5) exchangeable electrode cover, (6) valve of transfer system, (7) turbo molecular pump, (8) butterfly valve, (9) mass spectrometer, (10) capacitance manometer. The axial distance between the electrode and the quartz window is $l_{e l}=60 \mathrm{~mm}$. Electrode and window are circular with a radius of $r_{e l}=65 \mathrm{~mm}$ The chamber has a radius of $r_{c h}=125 \mathrm{~mm}$ and the total height is $l_{c h}=360 \mathrm{~mm}$.

flux ratios of $\mathrm{Ar}$ and $\mathrm{H}_{2}$ for the applied experimental conditions. The actual $\mathrm{Ar}$ and $\mathrm{H}_{2}$ partial pressures used in the experiment were, therefore, determined with a mass spectrometer ${ }^{22}$ prior to plasma ignition. These values do not differ significantly from the values measured after plasma ignition ${ }^{22}$.

The electron energy distribution function (EEDF), the electron temperature $T_{e}$, and the electron density $n_{e}$ were determined by a single-tip Langmuir probe system ${ }^{22} . T_{e}$ is calculated by the potential difference $\Delta V=V_{p l}-V_{f l} \operatorname{method}^{22}$, where $V_{p l}$ and $V_{f l}$ denote the plasma and the floating potential, respectively and $n_{e}$ is calculated by integrating the $\mathrm{EEDF}^{22}$. The Langmuir probe measurements were performed in the center of the plasma $(r=0 \mathrm{~mm}), 20 \mathrm{~mm}$ above the quartz window. For selected plasma conditions radiallyresolved measurements were conducted. In a pure $\mathrm{H}_{2}$ plasma at $1.5 \mathrm{~Pa}$ the electron density is constant for $0 \leq r \leq 30 \mathrm{~mm}$. Between $r=30 \mathrm{~mm}$ and $r=r_{e l}=65 \mathrm{~mm} n_{e}$ decreases monotonically to about $1 / 3$ and for $r>r_{e l}$ it remains approximately constant. The electron temperature has a maximum at $r=10 \mathrm{~mm}$. Between $r=10 \mathrm{~mm}$ and $r=r_{e l} T_{e}$ decreases by about $30 \%$ and shows a further slight decrease for larger $r$. In order to compare calculated absolute spectroscopy signals with measured ones weighting coefficients are calculated for $n_{e}$ and $T_{e}$ to account for the radial profiles. The weighting coefficients are used to convert the values measured in the center of the plasma (i.e., at $r=0 \mathrm{~mm}$ ) to line-of-sight averaged values. These weighting coefficients of $T_{e}$ and $n_{e}$ are 1.0 and 0.7 , respectively. These line-ofsight averaged values of $T_{e}$ and $n_{e}$ are then used to calculate absolute spectroscopy signals (see Sec. III) using a 0-dimensional model. 


\begin{tabular}{cccc} 
line & species & $\lambda_{0}$ & $\Delta \lambda$ \\
$(\mathrm{nm})$ & $(\mathrm{nm})$ \\
\hline $\mathrm{Ar}_{750}$ & $\mathrm{Ar}$ & 750.39 & 0.63 \\
$\mathrm{H}_{\beta}$ & $\mathrm{H}$ & 486.13 & 0.55 \\
$\mathrm{H}_{\gamma}$ & $\mathrm{H}$ & 434.02 & 0.58 \\
$\mathrm{H}_{2} V_{22}^{F u l}\left(Q_{1}\right)$ & $\mathrm{H}_{2}$ & 622.48 & 0.44
\end{tabular}

TABLE I. Studied lines used for optical emission spectroscopy with corresponding wavelength $\lambda$ and the experimental line width $\Delta \lambda . \Delta \lambda$ is mostly determined by the experimental apparatus function.

\section{B. Optical emission spectroscopy}

OES was applied to measure the gas temperature and the dissociation degree of hydrogen. The used spectrometer is a Czerny-Turner spectrograph (Acton SpectraPro 275) with a focal length of $275 \mathrm{~mm}$. The experiments were conducted with a 1800 lines $/ \mathrm{mm}$ grating, which has a measured resolution of $0.15 \mathrm{~nm}$ at $\lambda=600 \mathrm{~nm}$. The entrance slit of the spectrometer has a width of $40 \mu \mathrm{m}$. The light is detected by a CCD array (EEV 256x1024 OE CCD30, PIXIS, Princeton Instruments). The measured signal $D(\lambda)$ (in arbitrary units) results from the emitted photons integrated along the line of sight through the plasma as a function of the wavelength $\lambda$ in a time interval $t_{i n t}$. The signal was relatively quantified by multiplying with the relative sensitivity curve $R(\lambda)$ obtained for our experimental setting using a calibrated halogen lamp and $\mathrm{D}_{2}$ arc discharge light source for calibration. To derive the experimental line intensity $\dot{N}^{m}$ of an emission line with the total line width $\Delta \lambda$ at the wavelength $\lambda_{0}$ the line profile is integrated:

$$
\dot{N}^{m} \propto \frac{1}{t_{\text {int }}} \int_{\lambda_{0}-\Delta \lambda / 2}^{\lambda_{0}+\Delta \lambda / 2}\left(D(\lambda)-D_{b g}(\lambda)\right) \times R(\lambda) d \lambda
$$

where $D_{b g}$ is the background signal which has to be subtracted. In this study the $\mathrm{H}_{\beta}$ line $(486.13 \mathrm{~nm})$ and the Ar line at $750.39 \mathrm{~nm}\left(\mathrm{Ar}_{750}\right)$ are used for actinometry. As measure of the intensity of the Fulcher- $\alpha$ transition the intensity $\dot{N}_{\mathrm{H}_{2} V_{22}^{F u l}\left(Q_{1}\right)}^{m}$ of the line $Q_{1}$ of the vibronic band $v^{6}=v^{*}=2$ at a wavelength of $622.48 \mathrm{~nm}$ is chosen. $\lambda_{0}$ and $\Delta \lambda$ of the studied lines are given in Table I.

The $\mathrm{H}_{2}$ gas temperature $T_{g}$ is derived from the rotational temperature $T_{\text {rot }}$ of the $\mathrm{H}_{2}$ molecule. $T_{\text {rot }}$ of the hydrogen molecule is derived from the rotational lines $Q_{1}-Q_{3}$ of the Q-branch of the $\mathrm{H}_{2}$ Fulcher- $\alpha$ diagonal band $\left(v^{6}=v^{\prime \prime}=2\right)$ with an experimental uncertainty of $50 \mathrm{~K}^{22} . T_{\text {rot }}$ is taken as an estimate of the gas temperature $T_{g}$ and is applied to calculate the gas densities according to the ideal gas law:

$$
n_{j}=f_{j} \frac{p}{k_{B} T_{g}}
$$

for the background gas $j$ with the fraction $f_{j}$ which denotes the partial pressure of species $j\left(k_{B}-\right.$ Boltzmann constant). 
Actinometry was used in this work to determine the $\mathrm{H}$ density. As actinomers $\mathrm{Ar}$ and $\mathrm{H}_{2}$ are used. In the following these two different methods will be called $\mathrm{Ar}$ and $\mathrm{H}_{2}$ actinometry. In the mixed plasma the results of the two actinometry methods $\left(\mathrm{Ar}\right.$ and $\left.\mathrm{H}_{2}\right)$ are compared with each other to check the validity of the $\mathrm{H}_{2}$ actinometry (see Sec III). The model adopted here is based on the simple corona equilibrium ${ }^{25}$. In steady state electron-impact excitation is counterbalanced by deexcitation through spontaneous emission to energetically lower lying states of the atom. The line intensity $\dot{N}_{i j}$ is the number of emitted photons at a certain wavelength $\lambda_{i j}$ arising from the transition from a state $i$ to a lower state $j$ which is irradiated per volume and time into the whole solid angle and can be expressed ${ }^{22}$ as function of the ground state density $n_{1}$ :

$$
\dot{N}_{i j}=n_{1} \times n_{e} \times K_{1 i}^{e f f}
$$

with the effective rate coefficient $K_{1 i}^{e f f}$ which is the product of the direct rate coefficient $K_{1 i}$ and the branching ratio $A_{i j} / \sum_{l<i} A_{i l}$. The rate coefficient $K_{1 i}$ is the product of the corresponding cross section and the electron velocity where this product is averaged over a Maxwell energy distribution of the electrons. The $\mathrm{H}_{\beta}$ line of atomic hydrogen at $486.1 \mathrm{~nm}$ is used because it is due to direct excitation from the ground state. Dissociative excitation of atomic hydrogen from molecular hydrogen can be neglected for the present plasma conditions, as shown in Ref. ${ }^{22}$ for comparable discharge conditions. The effective rate coefficient is taken directly from Ref. ${ }^{26}$. For the Ar atom the line at $750.4 \mathrm{~nm}$ is used because this line is also predominantly excited by direct excitation from the ground state ${ }^{27}$. The cross section is taken from Ref. ${ }^{28}$. The branching ratio $A_{i j} / \sum_{k<i} A_{i k}$ for this excited state is 1 (see for example Ref. ${ }^{29}$ ). The atomic hydrogen density is calculated applying Eqs. 2 and 3 and the measured line intensities $\dot{N}_{\mathrm{H}_{\beta}}$ and $\dot{N}_{\mathrm{Ar}_{750}}$. The ratio of the atomic, $n_{\mathrm{H}}$, to molecular hydrogen density, $n_{\mathrm{H}_{2}}$, which is called dissociation degree in the following, is given by (Ar actinometry):

$$
\frac{n_{\mathrm{H}}}{n_{\mathrm{H}_{2}}}=\frac{\dot{N}_{\mathrm{H}_{\beta}} \times K_{\mathrm{Ar}_{750}}^{e f f} \times n_{e} \times n_{\mathrm{Ar}}}{\dot{N}_{\mathrm{Ar}_{750}} \times K_{\mathrm{H}_{\beta}}^{e f f} \times n_{e}} \times \frac{1}{n_{\mathrm{H}_{2}}}=\frac{\dot{N}_{\mathrm{H}_{\beta}} \times K_{\mathrm{Ar}_{750}}^{e f f}}{\dot{N}_{\mathrm{Ar}_{750}} \times K_{H_{\beta}}^{e f f}} \times \frac{f_{\mathrm{Ar}}}{1-f_{\mathrm{Ar}}} .
$$

The experimental uncertainty for the dissociation degree (Eqn. 4) is $24 \%$. The following uncertainties contribute: counting statistics of the spectroscopic signal $D(<4 \%$, more than 1000 counts for each measurement), reproducibility (10\%), error of effective rate coefficient ratio $K_{\mathrm{Ar}_{750}}^{e f f} / K_{\mathrm{H}_{\beta}}^{e f f}(13 \%)$ which is a function of the error in electron temperature, and the error of the calibration curve $R(\lambda)(18 \%)$ are considered ${ }^{22}$.

In addition to the actinometric measurements in Ar admixed plasmas, $n_{\mathrm{H}}^{m} / n_{\mathrm{H}_{2}}$ is determined from the ratio of $\dot{N}_{\mathrm{H}_{\gamma}}^{m}$ and $\dot{N}_{\mathrm{H}_{2}^{F u l}}^{m}$. Here, $\dot{N}_{\mathrm{H}_{2}^{F u l}}^{m}$ denotes the relative line intensity of the total Fulcher- $\alpha$ transition considering all rotational and vibrational lines. In fact, only the first 5 lines $\left(J^{*}=1-5\right)$ in the Q- branch of $V_{00}^{F u l}, V_{11}^{F u l}, V_{22}^{F u l}$, and $V_{33}^{F u l}$ (here $V_{v^{v} v^{*}}^{F u l}$ denotes the vibrational transition from the higher vibrational level $v^{6}$ to the lower one $v$ " within the Fulcher- $\alpha$ transition) are measured because the other lines have very low intensities for the considered conditions. $V_{00}^{F u l}-V_{33}^{F u l}$ are in the wavelength range between 600 and $640 \mathrm{~nm}$. The conversion of the measured data to $\dot{N}_{\mathrm{H}_{2}^{F}}^{m}$ is complex and for details the reader 


\begin{tabular}{ccccc}
$\begin{array}{c}f_{\mathrm{Ar}} \\
(\%)\end{array}$ & $\begin{array}{c}P_{r f} \\
(\mathrm{~W})\end{array}$ & $\begin{array}{c}n_{e} \\
\left(10^{16} \mathrm{~m}^{-3}\right)\end{array}$ & $\begin{array}{c}T_{e} \\
(\mathrm{eV})\end{array}$ & $\begin{array}{c}T_{\text {rot }} \\
(\mathrm{K})\end{array}$ \\
\hline 0 & 235 & 0.7 & 4.2 & 430 \\
12 & 165 & 1.0 & 3.7 & 450 \\
29 & 145 & 1.6 & 3.5 & 470
\end{tabular}

TABLE II. Plasma conditions $\left(f_{\mathrm{Ar}}-\mathrm{Ar}\right.$ fraction, $P_{r f}-r f$ input power $)$ and experimental results $\left(n_{e}\right.$ - electron density, $T_{e}$ - electron temperature from Langmuir probe measurements, $T_{\text {rot }}$ - rotational temperature of the Fulcher- $\alpha\left(v^{6}=v^{*}=2\right)$ transition) of the studied $\mathrm{H}_{2}$-Ar plasma for a total pressure of $1.5 \mathrm{~Pa}$.

is referred to the work of Manhard ${ }^{30}$. Here, the conversion will be outlined qualitatively in brief. Primarily, the rotational temperatures of the respective vibrational transition of the excited levels are calculated from the measured lines $J^{\natural}=1-5$ (see also Ref. ${ }^{22}$ ). The relative line intensity of a rotational line is a function of the rotational temperature and molecular constants. To yield the total line intensity of the $\mathrm{P}, \mathrm{Q}$, and $\mathrm{R}$ branch of the respective vibra-

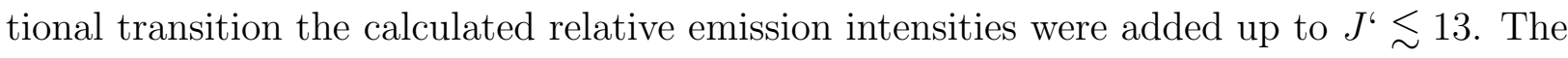
thus obtained relative line intensities of the $\mathrm{P}, \mathrm{Q}$ and $\mathrm{R}$ branch of a vibrational transition are added and multiplied by the measured $Q_{1}$ line intensity of the considered vibrational transition to obtain the total intensity of one vibrational transition. The sum of these four values is multiplied by a scaling factor $f_{v i b}=2.2$ which yields the total Fulcher transition line intensity $\dot{N}_{\mathrm{H}_{2}^{F u l}}^{m}$. $f_{v i b}$ accounts for the ratio of the line intensities of all possible vibrational transitions to the considered line intensities, i.e., from $V_{00}^{F u l}-V_{33}^{F u l}$. The thus obtained $\dot{N}_{\mathrm{H}_{2}^{F u l}}^{m}$ can now be used to determine the hydrogen dissociation degree $\left(\mathrm{H}_{2}\right.$ actinometry):

$$
\frac{n_{\mathrm{H}}}{n_{\mathrm{H}_{2}}}=\frac{\dot{N}_{\mathrm{H}_{\gamma}}^{m} \cdot K_{\mathrm{H}_{2}^{F u l}}^{e f f}}{\dot{N}_{\mathrm{H}_{2}^{F u l}}^{m} \cdot K_{\mathrm{H}_{\gamma}}^{e f f}} .
$$

Here, the $\mathrm{H}_{\gamma}$ line is used because $K_{\mathrm{H}_{2}^{\text {Ful }}}^{\text {eff }} / K_{\mathrm{H}_{\gamma}}^{\text {eff }}$ varies only slightly with $n_{e}$ and $T_{e}^{30}$. $K_{\mathrm{H}_{2}^{\text {Ful }}}^{\text {eff }}$ is calculated using a collisional-radiative model ${ }^{31}$. The ratio of the effective rate coefficients is 1.6 which is valid for $T_{e}=4 \mathrm{eV}$ and $n_{e}$ in the range of $10^{16} \mathrm{~m}^{-3}$ (see Ref. ${ }^{32}$ ).

\section{RESULTS}

\section{A. Emission intensities}

In the following results for measured relative line intensities are shown as function of $f_{\text {Ar. }}$. The density of atomic hydrogen is derived from these line intensities. The investigated plasma conditions and the measured plasma parameters are listed in Table II. The plasma parameters were measured for the actual experimental conditions as described in Sec. II. $T_{e}$ decreases from $4.7 \mathrm{eV}$ for pure $\mathrm{H}_{2}$ to $3.5 \mathrm{eV}$ for $f_{\mathrm{Ar}}=29 \%$. Increasing the Ar fraction at constant $r f$ input power caused a strong increase of the plasma density. Therefore, the 


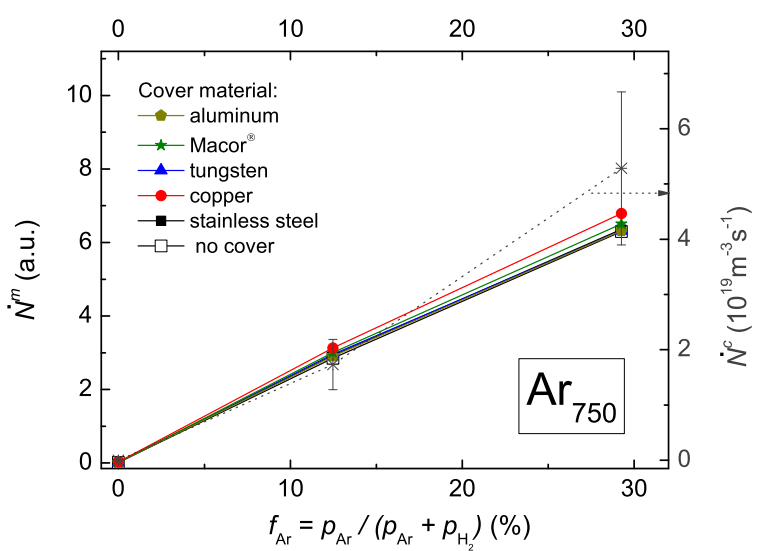

FIG. 2. Measured relative line intensity $\dot{N}^{m}$ (left-hand scale) of the $\operatorname{Ar}_{750}$ line for different materials of the electrode cover in a $\mathrm{H}_{2}$-Ar plasma as function of $f_{\mathrm{Ar}}$ for a total pressure of $1.5 \mathrm{~Pa}$. The calculated absolute line intensity $\dot{N}^{c}$ is shown by the dotted curve (right-hand scale). Error bars according to the counting statistics of the measured values are comparable to the symbol sizes. For the error of the calculated values see text.

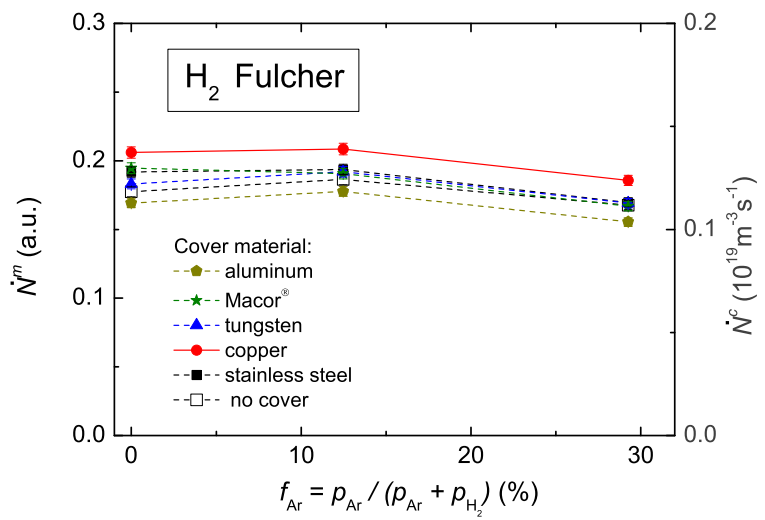

FIG. 3. Measured relative line intensity $\dot{N}^{m}$ of the $Q_{1}$ line of the vibronic band $v^{6}=v^{\prime \prime}=2$ of the $\mathrm{H}_{2}$ Fulcher- $\alpha$ transition for different materials of the electrode cover in a $\mathrm{H}_{2}$-Ar plasma as function of $f_{\text {Ar }}$ for a total pressure of $1.5 \mathrm{~Pa}$. Error bars according to the counting statistics are comparable to the symbol sizes. On the right-hand scale the absolute line intensity is shown. The required calibration constant was determined from the line intensity of the $\mathrm{Ar}_{750}$ line (for details, see text).

$r f$ power was adjusted in order to minimize the variation in $n_{e} . n_{e}$ was maintained in the range between $0.7 \times 10^{16} \mathrm{~m}^{-3}$ and $1.6 \times 10^{16} \mathrm{~m}^{-3}$ for the studied conditions. The measured $T_{\text {rot }}$ increases from $(430 \pm 50) \mathrm{K}$ for pure $\mathrm{H}_{2}$ to $(470 \pm 50) \mathrm{K}$ for $f_{\mathrm{Ar}}=29 \%$. For simplicity, in the following a value of $(450 \pm 50) \mathrm{K}$ is used as estimate for the gas temperature $T_{g}$.

In order to determine the influence of the material on the plasma parameters $n_{e}, T_{e}$, and 
$n_{\mathrm{H}}$, the relative line intensities $\dot{N}_{\mathrm{H}_{\beta}}^{m}$ of atomic hydrogen, $\dot{N}_{\mathrm{H}_{2} V_{22}^{F u l}\left(Q_{1}\right)}^{m}$ of molecular hydrogen, and $\dot{N}_{\mathrm{Ar} 750}^{m}$ of the Ar atom are measured. According to the corona model the line intensities are proportional to the ground state density of the corresponding species as well as to $n_{e}$ and $K^{e f f}\left(T_{e}\right)$ (see Eq. 3). The measured relative line intensities $\dot{N}_{\mathrm{Ar}_{750}}^{m}$ of the $\operatorname{Ar}_{750}$ line as well as the calculated line intensity $\dot{N}_{\mathrm{Ar} 750}^{c}$ using Eq. 3 are shown in Fig. 2 as a function of $f_{\text {Ar }}$ for different materials of the electrode cover. $\dot{N}_{\mathrm{Ar}_{750}}^{m}$ increases linearly with $f_{\mathrm{Ar}}$. For all investigated materials the measured argon line intensities are almost identical. According to Eq. $3 \dot{N}_{\mathrm{Ar} 750}^{c}$ is the product of $n_{\mathrm{Ar}}=f_{\mathrm{Ar}} \times p \times\left(k_{B} T_{g}\right)^{-1}, n_{e}$, and $K_{\mathrm{Ar}_{750}}^{\text {eff }}\left(T_{e}\right)$, where $T_{g}=450 \mathrm{~K}$ and the data from Table II are used. The relative experimental uncertainty of the calculated line intensity $\dot{N}_{\mathrm{Ar}_{750}}^{c}$ comprises four contributions: the uncertainty of the measured electron density (relative error of $15 \%$ ), rate coefficient (18\% which is based on a relative error of $T_{e}$ of $6 \%$, taking only into account the reproducibility), Ar fraction (5\%), and gas temperature (11\%). A total uncertainty of $26 \%$ is obtained by Gaussian error propagation. The calculated $\dot{N}_{\mathrm{Ar} 750}^{c}$ does not increase linearly with $f_{\text {Ar }}$ because the measured $n_{e}$ and $T_{e}$ values vary with $f_{\mathrm{Ar}}$. Nevertheless, the experimental data and the model results of $\dot{N}_{\mathrm{Ar}_{750}}$ shown in Fig. 2 agree within the experimental uncertainty of $\dot{N}_{\mathrm{Ar}_{750}}^{c}$. The calculated $\dot{N}_{\mathrm{Ar}_{750}}$ is the number of emitted photons per volume and time. From the comparison between model and measurement a calibration constant can be determined which quantifies $\dot{N}_{\mathrm{Ar}_{750}}^{m}$ absolutely. Since $\dot{N}_{\mathrm{Ar}_{750}}^{m}$ and the line intensities $\dot{N}_{\mathrm{H}_{2} V_{22}^{F u l}\left(Q_{1}\right)}^{m}$ and $\dot{N}_{\mathrm{H}_{\beta}}^{m}$ in Figs. 3 and 4 are calibrated relatively to each other (see Sec. II and Eq. 1), $\dot{N}_{\mathrm{H}_{2} V_{22}^{F u l}\left(Q_{1}\right)}^{m}$ and $\dot{N}_{\mathrm{H}_{\beta}}^{m}$ are also absolutely calibrated by this procedure. In Fig. 3 the values of $\dot{N}_{\mathrm{H}_{2} V_{22}}^{m}{ }^{F u}\left(Q_{1}\right)$ are shown. They vary only slightly with varying $f_{\mathrm{Ar}}$ and varying electrode cover material. Other Fulcher lines, i.e., $Q_{1}$ of $v^{6}=v^{*}=0,1,3$, which are not shown here, do also not differ significantly for different electrode cover materials.

The fact that $\dot{N}_{\mathrm{H}_{2} V_{22}^{F u l}\left(Q_{1}\right)}^{m}$ in Fig. 3 is more or less independent of $f_{\mathrm{Ar}}$ is surprising. Increasing $f_{\text {Ar }}$ to $29 \%$ should result in a decrease of the Fulcher band intensity because the $\mathrm{H}_{2}$ density decreases. However, the decrease in the $\mathrm{H}_{2}$ density is obviously compensated by the increase of the electron density (see also Table II).

The independence of the line intensities for both $\mathrm{Ar}$ and $\mathrm{H}_{2}$ from the used electrode cover materials indicates that the plasma parameters $n_{e}, T_{e}$, and $T_{g}$ do not differ noticeably from each other for different electrode cover materials, since the line intensities are a sensitive function of $n_{e}, T_{e}$, and $T_{g}$ (see Eq. 3).

Finally, in Fig. 4 the measured line intensities $\dot{N}_{\mathrm{H}_{\beta}}^{m}$ of the $\mathrm{H}_{\beta}$ lines are presented as a function of $f_{\mathrm{Ar}}$ for different materials of the cover electrode. The $\dot{N}_{\mathrm{H}_{\beta}}^{m}$ values for the different electrode cover materials are largely different. The lowest line intensities are observed for electrode covers made of stainless steel and copper. Significantly larger line intensities are observed for tungsten and Macor ${ }^{\circledR}$ and the highest line intensity is observed for aluminum. The values for the same material do not differ noticeably with increasing $f_{\text {Ar }}$ in the considered $f_{\text {Ar }}$ range. In this respect it should be noted that for different $f_{\text {Ar }}$ a constant $\dot{N}_{\mathrm{H}_{\beta}}^{m}$ does not necessarily result in a constant atomic hydrogen density because $n_{e}$ and $T_{e}$ do also change with varying $f_{\mathrm{Ar}}$ (see Eq. 3). To test the possible disturbance of the plasma by introducing the electrode cover the line intensities of the measurement in which the electrode made 


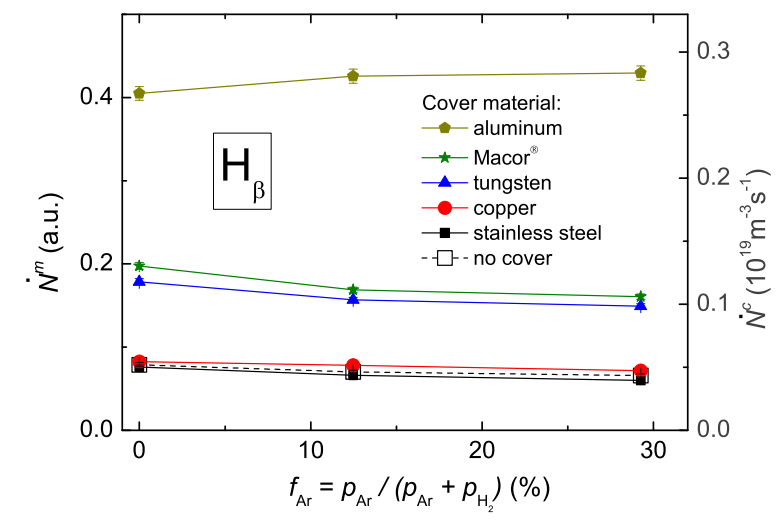

FIG. 4. Measured relative line intensity $\dot{N}^{m}$ of the $\mathrm{H}_{\beta}$ line for different materials of the cover electrode in a $\mathrm{H}_{2}$-Ar plasma as function of $f_{\mathrm{Ar}}$ for a total pressure of $1.5 \mathrm{~Pa}$. Error bars according to the counting statistics are comparable to the symbol sizes. On the right-hand scale the absolute line intensity is shown. The required calibration constant was determined from the line intensity of the $\mathrm{Ar}_{750}$ line (for details, see text).

of stainless steel is not covered is compared with the measurement where the electrode cover made of stainless steel is used. These two measurements are within the experimental uncertainty of about $4 \%$ identical. This indicates that the surface conditions of the electrode and the electrode cover are also identical. The measurement with copper shows within the experimental uncertainty the same line intensity as the measurement with stainless steel. The individual measurements with the various electrode cover materials are carried out on different days. Due to cooling and warming of the electrode it could be that the conditions on the surface of the materials and, therefore, $\dot{N}_{\mathrm{H}_{\beta}}^{m}$ have changed. Such an influence of the electrode temperature has also been observed for the energy influx measured in an $\mathrm{H}_{2}-\mathrm{Ar}$ plasma $^{33}$. Repeated measurements with copper have shown that the line intensities are within the experimental uncertainty identical. Therefore, equal conditions on the surface of the materials in different experiments are assumed.

\section{B. Atomic hydrogen density}

The dissociation degree $n_{\mathrm{H}}^{m} / n_{\mathrm{H}_{2}}$ measured by Ar actinometry (see Eq. 4) is shown in Fig. 5. Within the experimental uncertainty $n_{\mathrm{H}}^{m} / n_{\mathrm{H}_{2}}$ is almost independent of $f_{\mathrm{Ar}}$. The same trend of $n_{\mathrm{H}}^{m} / n_{\mathrm{H}_{2}}$ as for $\dot{N}_{\mathrm{H}_{\beta}}^{m}$ is obtained if the different materials of the electrode cover are considered: The values of $n_{\mathrm{H}}^{m} / n_{\mathrm{H}_{2}}$ for stainless steel and copper are the lowest, the values for tungsten and Macor ${ }^{\circledR}$ are higher by about a factor of two, and the values for aluminum are higher by a factor of about five than the one for stainless steel, respectively.

In addition to $\mathrm{Ar}$ actinometry, $n_{\mathrm{H}}^{m} / n_{\mathrm{H}_{2}}$ is determined from $\mathrm{H}_{2}$ actinometry, too. In Fig. 5 $n_{\mathrm{H}}^{m} / n_{\mathrm{H}_{2}}$ from $\mathrm{H}_{2}$ actinometry is also shown for the electrode cover materials stainless steel, tungsten, and aluminum. The corresponding data for copper and Macor ${ }^{\circledR}$ are not shown but they agree within the experimental uncertainty with the data for stainless steel and tungsten, 


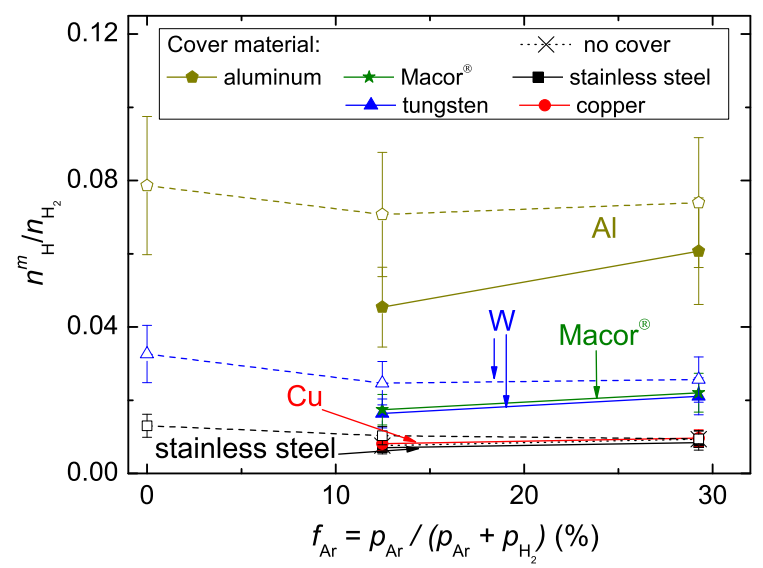

FIG. 5. Measured dissociation degree $n_{\mathrm{H}}^{m} / n_{\mathrm{H}_{2}}$ of hydrogen obtained with actinometry for different materials of the cover electrode in a $\mathrm{H}_{2}$-Ar plasma as a function of $f_{\mathrm{Ar}}$ for a total pressure of $1.5 \mathrm{~Pa}$. As actinomer $\mathrm{Ar}$ (closed symbols) and $\mathrm{H}_{2}$ (open symbols) were used, respectively. The experimental uncertainty of $n_{\mathrm{H}}^{m} / n_{\mathrm{H}_{2}}$ is $24 \%$ (see text).

respectively. Where both methods were applied the results from Ar actinometry agree within the experimental uncertainty with those obtained from $\mathrm{H}_{2}$ actinometry for the identical material. Therefore, we conclude that the $\mathrm{H}_{2}$ actinometry also provides reliable results. For the sake of completeness, the absolute hydrogen densities $n_{\mathrm{H}}$ are given in Table III for the different materials and gas mixtures. The $n_{\mathrm{H}}$ values in Table III are calculated by applying Eq. 2 for a gas temperature of $450 \mathrm{~K}$ and an atomic hydrogen fraction $f_{\mathrm{H}}$ which is the product of the molecular hydrogen fraction $f_{\mathrm{H}_{2}}=1-f_{\mathrm{Ar}}$ and the dissociation degree of Fig. 5. The most important result is that for different electrode cover materials $n_{\mathrm{H}}$ changes. The hydrogen density ratios of two electrode cover materials are exactly the dissociation degree ratios of these two electrode cover materials for the same plasma condition (same pressure, $r f$ power, and Ar fraction). Moreover, the $\mathrm{H}$ density ratio of two electrode cover materials is nearly independent of the Ar fraction.

\section{DISCUSSION}

\section{A. Model for the wall loss}

In low-pressure $\mathrm{H}_{2}$ - $\mathrm{Ar}$ plasmas with low $\mathrm{Ar}$ content the $\mathrm{H}$ atoms are produced almost exclusively from the electron-induced dissociation of $\mathrm{H}_{2}$ (reaction $\mathrm{e}^{-}+\mathrm{H}_{2} \rightarrow 2 \mathrm{H}+\mathrm{e}^{-}$) with the corresponding rate coefficient $K_{\text {diss }}$ and are lost to the wall. At the wall $\mathrm{H}$ atoms recombine forming $\mathrm{H}_{2}$. Other loss processes such as three-body recombination in the plasma volume or pumping can be neglected for the present conditions ${ }^{14-17,34}$. The wall loss time $t_{w \mathrm{H}}$, which is the inverse of the wall loss rate coefficient, is the mean time for a hydrogen atom to reach the plasma surrounding wall. The $\mathrm{H}$ atom density is then given by the continuity 


\begin{tabular}{c|c|c|c} 
& \multicolumn{3}{|c}{$n_{\mathrm{H}}\left(10^{18} \mathrm{~m}^{-3}\right)$} \\
\hline Electrode cover material & $f_{\mathrm{Ar}}=0 \%$ & $f_{\mathrm{Ar}}=12 \%$ & $f_{\mathrm{Ar}}=29 \%$ \\
stainless steel & 3.4 & 1.5 & 1.4 \\
copper & 3.3 & 1.6 & 1.6 \\
tungsten & 7.8 & 3.5 & 3.6 \\
Macor ${ }^{\circledR}$ & 8.3 & 3.7 & 3.8 \\
aluminum & 19 & 9.6 & 10
\end{tabular}

TABLE III. Absolute atomic hydrogen densities $n_{\mathrm{H}}$ for different materials of the electrode cover and different argon fractions $f_{\mathrm{Ar}} . n_{\mathrm{H}}$ for $f_{\mathrm{Ar}}=0 \%$ are measured by $\mathrm{H}_{2}$ Fulcher actinometry only. $n_{\mathrm{H}}$ for $f_{\mathrm{Ar}}=12$ and $29 \%$ are obtained from Ar actinometry which agree within the experimental uncertainty with the corresponding values of the $\mathrm{H}_{2}$ Fulcher actinometry. The total pressure of the $\mathrm{H}_{2}$-Ar plasma was 1.5 Pa.

equation:

$$
\frac{\partial n_{\mathrm{H}}}{\partial t}=2 n_{\mathrm{H}_{2}} \times n_{e} \times K_{\text {diss }}\left(T_{e}\right)-\frac{n_{\mathrm{H}}}{t_{w \mathrm{H}}} .
$$

In steady state Eq. 6 yields for the wall loss time of atomic hydrogen:

$$
t_{w \mathrm{H}}=\frac{n_{\mathrm{H}}}{n_{\mathrm{H}_{2}}} \times \frac{1}{2 n_{e} \times K_{\text {diss }}\left(T_{e}\right)} .
$$

In general, $t_{w \mathrm{H}}$ can be described by a diffusive part $t_{D \mathrm{H}}$ and a surface loss part $t_{s \mathrm{H}}{ }^{25,35}$ :

$$
t_{w \mathrm{H}}=t_{D \mathrm{H}}+t_{s \mathrm{H}}
$$

The diffusion time of atomic hydrogen is given by ${ }^{17,35}$ :

$$
t_{D \mathrm{H}}=\frac{\Lambda^{2}}{D_{\mathrm{H}}}
$$

( $\Lambda$ - diffusion length, $D_{\mathrm{H}}$ - diffusion constant) and the surface loss time is given by ${ }^{17,35}$ :

$$
t_{s \mathrm{H}}=\frac{V}{A} \frac{2\left(2-\beta_{\mathrm{H}}\right)}{\beta_{\mathrm{H}}} \frac{1}{v_{\mathrm{H}}}
$$

( $V$ - plasma volume, $A$ - wall area for loss of $\mathrm{H}, \beta_{\mathrm{H}}$ - surface loss probability for $\mathrm{H}, v_{\mathrm{H}}=$ $\sqrt{8 k_{B} T_{\mathrm{H}} / \pi M_{\mathrm{H}}}$ - mean velocity of $\mathrm{H}, M_{\mathrm{H}}$ - mass of $\mathrm{H}, T_{\mathrm{H}}$ - temperature of $\left.\mathrm{H}\right)$.

In our experiment the plasma interacts with two chamber wall materials: stainless steel (electrode) and quartz (dielectric window). Quartz has a very low $\beta_{\mathrm{H}}$ around $0.001^{16,36}$ and stainless steel a $\beta_{\mathrm{H}}$ in the range of $0.1^{15,19,36}$. Therefore, it is assumed that atomic hydrogen is only lost at the electrode (see also the preceding publication ${ }^{17}$ ) and the respective area for loss of $\mathrm{H}$ is $A=\pi r_{e l}^{2}$.

For further analysis it has to be kept in mind that the cover electrode only covers $84 \%$ of the area of the electrode. The possible influence of these uncovered lateral surfaces on 


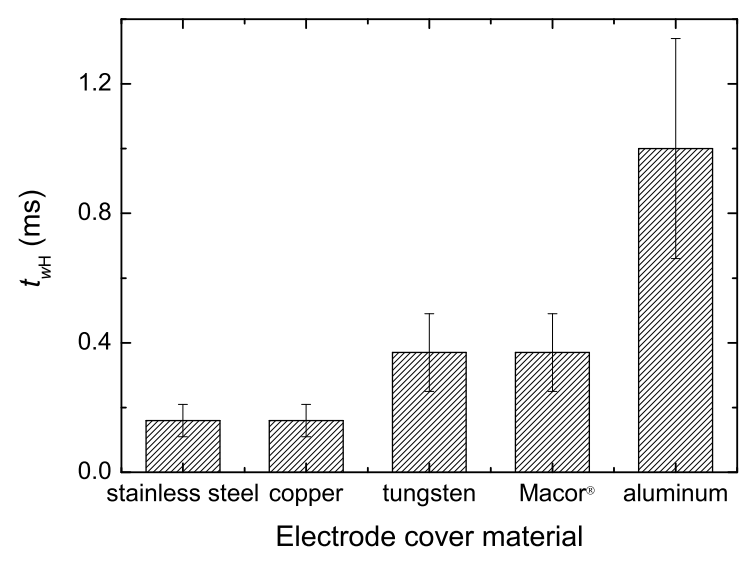

FIG. 6. Wall loss time $t_{w \mathrm{H}}$ derived from measured dissociation degrees for different materials of the cover electrode. The total pressure was $1.5 \mathrm{~Pa}$ and the Ar fraction was $29 \%$.

the measured $\mathrm{H}$ density will be discussed in the following. The line-of-sight of the emission spectroscopy runs along the axis which is parallel to the long side of the cover electrode (see Fig. 1). This means that the uncovered lateral surfaces are relatively far away from the line of sight. For a mean free path length of atomic hydrogen which is for our conditions comparable to the distance $l_{e l}$ between electrode and quartz window the loss of atomic hydrogen and, therefore, the local $\mathrm{H}$ density is mainly influenced by the nearest electrode surface area of the electrode. The probability of a particle, which starts in the radial center $(r=0 \mathrm{~mm}) 20 \mathrm{~mm}$ above the quartz window, to impinge on the uncovered lateral surfaces of the electrode is estimated by a simple calculation. The impact probability is assumed to be equal to the ratio of the solid angle of the uncovered lateral surfaces to the full solid angle of $4 \pi$. This implicitly includes the assumption that the particles do not collide with the background gas and are lost only at the surface. The probability of an $\mathrm{H}$ atom produced at $r=0 \mathrm{~mm}$ to impinge on the uncovered area is $6 \%$. For other positions along the line of sight this probability is even lower. Due to this low probability it is assumed that the influence of the uncovered lateral surface on the result of $\beta_{\mathrm{H}}$ is in our case negligible.

In the following the wall loss time $t_{w \mathrm{H}}$ is derived from the measured dissociation degree. In Sec. III it was shown that the measured line intensity $\dot{N}_{\mathrm{H}_{\beta}}^{m}$ varies for different materials of the cover electrode whereas $n_{e}, T_{e}$, and $T_{g}$ are essentially constant. According to the corona model $\left(\dot{N}_{\mathrm{H}_{\beta}}^{m} \propto n_{\mathrm{H}}\right.$, see Eq. 3) the change of $\dot{N}_{\mathrm{H}_{\beta}}^{m}$ can then be attributed solely to a change of $n_{\mathrm{H}}$. According to Eq. $6 n_{\mathrm{H}}$ is a function of $n_{e}, T_{e}$, and $t_{w \mathrm{H}}$. Since $n_{e}$ and $T_{e}$ do not differ significantly for different materials, the change of $n_{\mathrm{H}}$ is solely attributed to a change of $t_{w \mathrm{H}}$. Therefore, the wall loss time $t_{w \mathrm{H}}$ can be calculated using Eq. 7. The dissociation degrees of the Ar actinometry for $f_{\mathrm{Ar}}=12$ and $29 \%$ are taken from Fig. 5 and $n_{e}$ and $T_{e}$ are taken from Table II. This yields (see also Fig. 6):

$t_{w \mathrm{H}}=(0.16 \pm 0.05) \mathrm{ms}$ for stainless steel (ss) and copper $(\mathrm{Cu})$,

$t_{w \mathrm{H}}=(0.37 \pm 0.12) \mathrm{ms}$ for tungsten $(\mathrm{W})$ and Macor ${ }^{\circledR}$,

$t_{w \mathrm{H}}=(1.0 \pm 0.34) \mathrm{ms}$ for aluminum $(\mathrm{Al})$. 
Given these values for $t_{w \mathrm{H}}$ the corresponding $\beta_{\mathrm{H}}$ 's can be derived: $t_{w \mathrm{H}}$ is the sum of $t_{D \mathrm{H}}$ and $t_{s \mathrm{H}} \cdot t_{s \mathrm{H}}$ is a function of the plasma chamber geometry, the hydrogen temperature, and of $\beta_{\mathrm{H}}$ (see Eq. 10). Since $\beta_{\mathrm{H}}$ is the only variable in Eqs. 8-10 that depends on the electrode cover material, a variation in $t_{w \mathrm{H}}$ for different cover electrode material is attributed to a variation in $\beta_{\mathrm{H}}$. A calculation of the diffusion time $t_{D \mathrm{H}}=\Lambda^{2} / D_{\mathrm{H}}$ confirms that $t_{D \mathrm{H}}$ can be neglected for the present conditions. For our geometry a diffusion length of $\Lambda=l_{e l} / \pi=1.9 \times 10^{-2} \mathrm{~m}$ and the diffusion constant of $D_{\mathrm{H}}=44 \mathrm{~m}^{2} \cdot \mathrm{s}^{-1}$ for a hydrogen temperature of $T_{\mathrm{H}}=T_{g}=450 \mathrm{~K}$, an Ar fraction of $f_{\mathrm{Ar}}=12 \%$, and a pressure of $p=1.5 \mathrm{~Pa}$ are used (see model and formalism of Ref. ${ }^{17}$ ). This yields a diffusion time of $t_{D \mathrm{H}}=0.008 \mathrm{~ms}$. For the lowest measured value of $t_{w \mathrm{H}}$ of $0.16 \mathrm{~ms}$ (stainless steel) the calculated $t_{D \mathrm{H}}$ corresponds to $5 \%$ of $t_{w \mathrm{H}}$. For the other materials it is significantly lower. Therefore, for the applied pressure of $1.5 \mathrm{~Pa}$ the wall loss time of atomic hydrogen is approximated to:

$$
t_{w \mathrm{H}} \approx t_{s \mathrm{H}}
$$

$\beta_{\mathrm{H}}$ can now be calculated from Eq. 10 and 11 using the measured $t_{w \mathrm{H}}$. To determine $\beta_{\mathrm{H}}$ from the wall loss time, $V / A$ and $T_{\mathrm{H}}$ have to be known. The volume to surface ratio was estimated in a preceding publication ${ }^{17}$ to $V / A=l_{e l}=0.06 \mathrm{~m}$.

In the studied $\mathrm{H}_{2}$-Ar plasma at $1.5 \mathrm{~Pa}$ and low $\mathrm{Ar}$ content the $\mathrm{H}$ atoms are produced almost exclusively from the electron-induced dissociation of $\mathrm{H}_{2}$ (see Ref. ${ }^{17}$ ) via the repulsive $\mathrm{H}_{2}\left(b^{3} \Sigma_{u}^{+}\right)$state. As consequence the hydrogen atoms will have a kinetic energy of about $3.5 \mathrm{eV}$ (see Refs. ${ }^{37}$ and $^{38}$ ) according to the Franck-Condon principle. $\mathrm{H}$ production by other dissociation mechanisms, e.g., $\mathrm{e}^{-}+\mathrm{H}_{3}^{+} \rightarrow 3 \mathrm{H}$ or $\mathrm{H}^{+}+$wall $\rightarrow \mathrm{H}$, can be neglected ${ }^{17}$. Dissociation mechanisms based on electron-induced excitation of $\mathrm{H}_{2}$ resulting into higher excited $\mathrm{H}_{2}$ states (higher than $\mathrm{H}_{2}\left(b^{3} \Sigma_{u}^{+}\right)$, see Ref. ${ }^{39}$ ) will be discussed in the following. $\mathrm{H}$ production via these higher excited states would end up in excited atomic hydrogen. This would increase considerably the measured intensity of $\mathrm{H}$ Balmer lines. However, a simple collisional radiative model has shown that the largest part of the $\mathrm{H}_{\beta}$ line originates from atomic hydrogen and not from molecular hydrogen ${ }^{22}$. A comparable consideration for the $\mathrm{H}_{\alpha}$ line gave the same result. Therefore, we conclude that production of atomic hydrogen via higher than $\mathrm{H}_{2}\left(b^{3} \Sigma_{u}^{+}\right)$excited states plays only a minor role.

The Franck-Condon energy of several eV of the dissociated hydrogen will be dissipated by collisions with the background gas which has a temperature of $T_{g}$. The degree to which this energy is dissipated depends on the background gas pressure and, hence, on the average number of collisions with background gas species. Therefore, the $\mathrm{H}$ temperature should be in the range between $3.5 \mathrm{eV}$ and $T_{g}$. Studies in different discharge types (mostly in microwave discharges) at considerably higher pressures $(\geq 30 \mathrm{~Pa})$ than in the present study have measured the atomic hydrogen temperature via line broadening in the range between 1000 and $3000 \mathrm{~K}^{37,38,40-42}$. The measured atomic hydrogen temperatures were considerably lower than $3.5 \mathrm{eV}$ but higher than the gas temperature. In an inductively-coupled plasma which is nearly identical (in geometry and wall materials) with the present one, Kadetov $^{23}$ measured the Doppler broadening of atomic hydrogen by two-photon absorption laser-induced fluorescence for nearly similar conditions of $2 \mathrm{~Pa}$ and $300 \mathrm{~W}$. The measured 
Doppler broadening corresponded to an atomic hydrogen temperature of $390 \mathrm{~K}$. The rotational temperature of the present study at $1.5 \mathrm{~Pa}$ and $235 \mathrm{~W}$, and $f_{\mathrm{Ar}}=0 \%$ obtained from molecular hydrogen is $T_{g}=430 \pm 50 \mathrm{~K}$. The rotational temperature in the present study and the atomic hydrogen temperature from Ref. ${ }^{23}$ obtained for almost similar plasma conditions agree within the experimental uncertainty. Furthermore, we observed in a recent study on atomic nitrogen which exhibits a behavior similar to atomic hydrogen (production by dissociation with excess kinetic energy in the eV range, loss to the wall, and comparable mean free path length of atomic nitrogen) that the temperature of atomic nitrogen is in the range of the gas temperature for the considered plasma conditions ${ }^{43}$. Therefore, it is assumed that the temperature of atomic hydrogen is equal to the gas temperature. The measurements of Kadetov showed the surprising result that $T_{\mathrm{H}}$ was more or less constant in the pressure range between 2 and $30 \mathrm{~Pa}$. Actually one would assume that $T_{\mathrm{H}}$ increases for decreasing pressure because the mean free path length of atomic hydrogen increases if the pressure decreases. For $2 \mathrm{~Pa}$ the mean free path length is about half of the distance between the electrodes $\left(l_{e l}=0.06 \mathrm{~m}\right)$. Atomic hydrogen which starts with an energy of about $3.5 \mathrm{eV}$ from dissociation would require many collisions with the background gas to reduce its kinetic energy in order to reach $T_{\mathrm{H}}=T_{g}$. But at a pressure of $2 \mathrm{~Pa}$ atomic hydrogen only collides a few times with the background gas which should be insufficient for thermalization. Summarizing, it remains an open question why atomic hydrogen shows a temperature equal to the gas temperature in measurements at these low pressures. Nevertheless, we continue with our evaluation assuming $T_{\mathrm{H}}=T_{g}$.

\section{B. Surface loss probability of atomic hydrogen}

With $T_{\mathrm{H}}=T_{g}=450 \mathrm{~K}$ the mean velocity of atomic hydrogen is $v_{\mathrm{H}}=3.2 \times 10^{3} \mathrm{~m} \cdot \mathrm{s}^{-1}$. Combining Eq. 10 and 11 with the values of $V / A, v_{\mathrm{H}}$, and the measured wall loss times, the resulting wall loss probabilities of $\mathrm{H}$ for the considered electrode cover materials are for the present conditions:

$$
\begin{gathered}
\beta_{\mathrm{H}}^{C u}=\beta_{\mathrm{H}}^{s s}=0.39 \pm 0.13, \\
\beta_{\mathrm{H}}^{W}=\beta_{\mathrm{H}}^{\text {Macor }}{ }^{\circledR}=0.19 \pm 0.06,
\end{gathered}
$$

and

$$
\beta_{\mathrm{H}}^{A l}=0.07 \pm 0.03
$$

However, as discussed above the atomic hydrogen temperature is only an estimate. If $T_{\mathrm{H}}$ would be set to $6,000 \mathrm{~K}(1,000 \mathrm{~K})$ the resulting $\beta_{\mathrm{H}}^{s s}$ would be $0.12(0.28)$.

The ratios $\beta_{\mathrm{H}}^{m 1} / \beta_{\mathrm{H}}^{m 2}$ of different electrode cover materials $m 1$ and $m 2$ can be directly correlated to the ratios of the measured $\mathrm{H}_{\beta}$ lines. Assuming in Eq. 10 the approximation $2-\beta \approx 2$ and combining Eqs. 10, 11, 7, and 4 this yields:

$$
\frac{\beta_{\mathrm{H}}^{m 1}}{\beta_{\mathrm{H}}^{m 2}}=\frac{\dot{N}_{\mathrm{H}_{\beta}^{m 2}}}{\dot{N}_{\mathrm{H}_{\beta}^{m 1}}},
$$


for a fixed discharge condition with constant $T_{e}, n_{e}$, and $f_{\mathrm{Ar}}$ (shown in Sec. III) for different materials of the electrode cover. In deriving Eq. 15 it is assumed that for a given plasma condition the atomic hydrogen temperature remains unchanged if different wall materials are used. The uncertainty of the ratio of the surface loss probability is identical to the relative uncertainty in the ratio of the measured relative $\mathrm{H}_{\beta}$ line intensities which is only due to counting statistics. Therefore, the uncertainty of $\beta_{\mathrm{H}}^{1} / \beta_{\mathrm{H}}^{2}$ is much lower $(\lesssim 6 \%)$ than that of the absolute $\beta_{\mathrm{H}}$ values (35\%). The resulting relative wall loss probabilities $\beta_{\mathrm{H}}^{m}$ of atomic hydrogen for various materials of the electrode cover referred to the value $\beta_{\mathrm{H}}^{s s}$ of stainless steel are:

$$
\begin{gathered}
\beta_{\mathrm{H}}^{C u}=\beta_{\mathrm{H}}^{s s}, \\
\beta_{\mathrm{H}}^{W}=\beta_{\mathrm{H}}^{\text {Macor }^{\circledR}}=\frac{\beta_{\mathrm{H}}^{s s}}{2.0}, \\
\beta_{\mathrm{H}}^{A l}=\frac{\beta_{\mathrm{H}}^{s s}}{5.3} .
\end{gathered}
$$

The here measured surface loss probability of $\mathrm{Al}$ is considerably lower than the value for stainless steel. This result could be caused by the presence of an oxide layer on top of the $\mathrm{Al}$ surface since dielectrica have, in general, a lower $\beta$ value than metals (see Ref. ${ }^{36,44}$ ). However, a $\mathrm{H}_{2}$-Ar plasma at $1.5 \mathrm{~Pa}\left(f_{\mathrm{Ar}}=29 \%\right)$ was operated for one hour before starting the measurements in order to reduce possible surface contaminations on the electrode surface (see Sec. II). This plasma treatment was carried out for all investigated electrode cover materials. We assume that oxide layers at the surfaces of the investigated electrode covers are reduced. We, therefore, suppose that the difference in $\beta_{\mathrm{H}}$ between $\mathrm{Al}$ and stainless steel is due to the different surface properties of $\mathrm{Al}$ and stainless steel. To clarify this assumption further investigations would be necessary.

Fantz ${ }^{31}$ studied in an inductively coupled plasma the influence of the materials steel, copper, aluminum and graphite on $n_{\mathrm{H}}$. $n_{\mathrm{H}}$ was determined by means of optical emission spectroscopy. Spatially resolved measurements above an additional surface in the plasma were carried out, but no $\beta_{\mathrm{H}}$ was derived. The result was that for aluminum $n_{\mathrm{H}}$ is approximately a factor of 3 higher than the values for stainless steel and copper. Graphite showed an even lower $n_{\mathrm{H}}$ than stainless steel and copper. Making the identical assumption as in our evaluation, namely that the plasma parameters and atomic hydrogen temperature do not change significantly, then the $\mathrm{H}$ density ratios of Ref. ${ }^{31}$ can be compared with our results. The fact that $n_{\mathrm{H}}$ for stainless steel and copper is identical is in excellent agreement with our result and the value for $n_{\mathrm{H}}^{A l} / n_{\mathrm{H}}^{s s}$ of 3 of Ref. ${ }^{31}$ is in fair agreement with our value of 5 .

The surface loss probability of atomic hydrogen for stainless steel or aluminum determined from plasma experiments is reported in several publications ${ }^{14,15,18-21}$. In publications ${ }^{14,15,18-20}$ the decay of the atomic hydrogen density in the afterglow of a capacitively coupled plasma with pure $\mathrm{H}_{2}$ feed gas was measured. Since volume recombination can be neglected the measured decay time is set equal to the wall loss time. The wall loss time is described by a model which is similar ${ }^{18,19}$ to or even identical ${ }^{14,15,20}$ to Eqs. 8-10. Finally, in these publications $\beta_{\mathrm{H}}$ was derived from the measured decay time. Mendez et al. ${ }^{21}$ studied a $\mathrm{H}_{2}$ DC plasma determining the density of atomic hydrogen by actinometry and by a rate equation model. The plasma reactor consisted of a stainless steel vessel. In accordance 


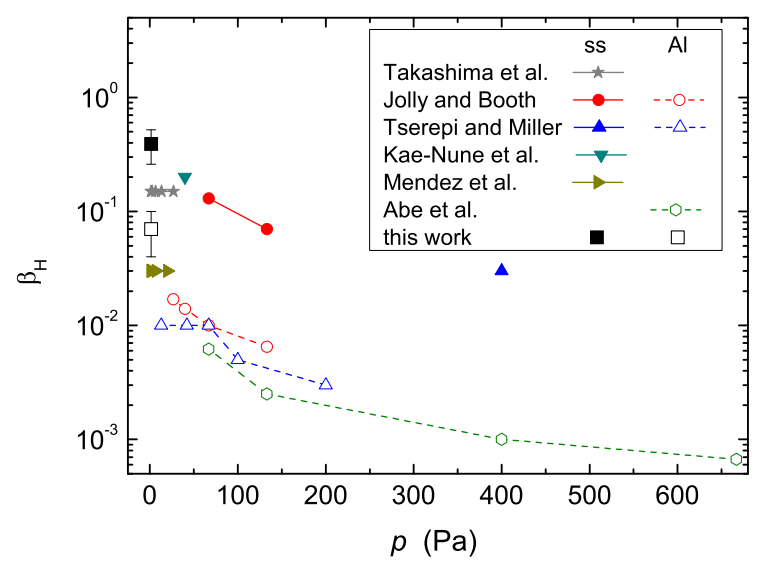

FIG. 7. Surface loss probability of atomic hydrogen for stainless steel and aluminum as function of the gas pressure $p$ from various references: Takashima et al. ${ }^{14}$, Jolly and Booth ${ }^{15}$, Tserepi and Miller ${ }^{18}$, Kae-Nune et al. ${ }^{19}$, Mendez et al. ${ }^{21}$, and Abe et al. ${ }^{20}$.

with the present work Mendez et al. found that atomic hydrogen is basically produced by dissociation of molecular hydrogen and lost by recombination at the wall. To achieve an agreement between calculated and measured $\mathrm{H}$ density a value of $\beta_{\mathrm{H}}$ of 0.03 was used. For all publications ${ }^{14,15,18-20}$ the temperature of atomic hydrogen was assumed to be equal to the gas temperature where values between 300 and $400 \mathrm{~K}$ were used. However, different authors studied $n_{\mathrm{H}}, t_{w \mathrm{H}}$, and, therefore, $\beta_{\mathrm{H}}$ for largely different plasma conditions. The most important parameter in their studies appears to be the gas pressure $p$. In Fig. $7 \beta_{\mathrm{H}}$ values for stainless steel and aluminum taken from the considered references ${ }^{14,15,18-20}$ are plotted as a function of $p$. $\beta_{\mathrm{H}}$ for stainless steel varies from 0.03 determined for a pressure of $400 \mathrm{~Pa}$ (see Ref. ${ }^{18}$ ) to 0.20 for $40 \mathrm{~Pa}$ (see Ref. ${ }^{19}$ ). $\beta_{\mathrm{H}}$ for aluminum varies from $6.7 \times 10^{-4}$ at $667 \mathrm{~Pa}$ (see Ref. ${ }^{20}$ and $^{45}$ ) to 0.017 at $27 \mathrm{~Pa}$ (see Ref. ${ }^{15}$ ). For low pressures Takashima et al. ${ }^{14}$ $\left(1 \leq p \leq 27 \mathrm{~Pa}, \beta_{\mathrm{H}}\right.$ for stainless steel $)$, Mendez et al. ${ }^{21}\left(0.8 \leq p \leq 20 \mathrm{~Pa}, \beta_{\mathrm{H}}\right.$ for stainless steel), and Tserepi and Miller ${ }^{18}\left(13 \leq p \leq 67 \mathrm{~Pa}, \beta_{\mathrm{H}}\right.$ for aluminum) obtained constant values for $\beta_{\mathrm{H}}$. $\beta_{\mathrm{H}}$ of stainless steel is about one order of magnitude higher than that of aluminum. The ratio of $\beta_{\mathrm{H}}^{A l} / \beta_{\mathrm{H}}^{s s}$ in the present work is 5.3 which is in moderate agreement with the literature. In Fig. 7 a general trend of a decreasing $\beta_{\mathrm{H}}$ values with increasing $p$ is observed. The reason for this trend is presently unknown. A possible explanation could be that for the experiments performed at higher pressure the surface temperature of the relevant surface areas is higher than at lower pressure and that $\beta_{\mathrm{H}}$ decreases with increasing substrate temperature. To clarify this issue further dedicated experiments would be required. The absolute $\beta_{\mathrm{H}}$ values determined in the present work for stainless steel $\left(\beta_{\mathrm{H}}^{s s}=0.39\right)$ and aluminum $\left(\beta_{\mathrm{H}}^{A l}=0.07\right)$ at a pressure of $1.5 \mathrm{~Pa}$ are in the same order of magnitude as the values published in Refs. ${ }^{14,15,18-20}$ but our values are clearly higher. It is assumed that these higher values are either due to the lower pressure compared to the conditions of the considered references or, as discussed above, due to the uncertainty of the determination of the atomic hydrogen temperature. 


\section{SUMMARY}

In an inductively-coupled $\mathrm{H}_{2}$-Ar plasma at a total pressure of $1.5 \mathrm{~Pa}$ the influence of the electrode materials stainless steel, copper, tungsten, Macor ${ }^{\circledR}$, and aluminum on the surface loss probability $\beta_{\mathrm{H}}$ of atomic hydrogen was determined by optical emission spectroscopy. Hydrogen dissociation degrees for the considered conditions were determined experimentally by actinometry using the emission intensity ratio of $\mathrm{H}_{\beta}$ and $\mathrm{Ar}_{750}$ (Ar actinometry) as well as the ratio of the $\mathrm{H}_{\gamma}$ and the $\mathrm{H}_{2}$ Fulcher $-\alpha$ transition $\left(\mathrm{H}_{2}\right.$ actinometry). The resulting dissociation degrees from $\mathrm{H}_{2}$ actinometry agree within the experimental uncertainties with those obtained from Ar actinometry. From the measured dissociation degrees $\beta_{\mathrm{H}}$ was determined assuming the mean temperature of the atoms $T_{\mathrm{H}}$ being equal to $T_{g}$. The absolute values of $\beta_{\mathrm{H}}$ for stainless steel and $\beta_{\mathrm{H}}$ for aluminum were found to be higher than previously published values. From a comparison of different published values it was concluded that measured $\beta_{\mathrm{H}}$ values decrease with increasing pressure. The values of this work are within this general trend.

The uncertainty in $n_{\mathrm{H}}$ leads to a large uncertainty in $\beta_{\mathrm{H}}$. However, the experimental uncertainty of ratios of different $\beta_{\mathrm{H}}$ values determined for comparable plasma conditions is considerably lower than that of the absolute values of $\beta_{\mathrm{H}}$. It was shown that the ratios of $\beta_{\mathrm{H}}$ values for different materials are the inverse of the measured emission intensity ratios of the $\mathrm{H}_{\beta}$ line. These ratios are in first order approximation independent of the atomic hydrogen temperature. Our results show that $\beta_{\mathrm{H}}$ of copper is equal to the value of stainless steel, $\beta_{\mathrm{H}}$ of Macor ${ }^{\circledR}$ and tungsten is about 2 times smaller and $\beta_{\mathrm{H}}$ of aluminum about 5 times smaller compared to stainless steel. The latter ratio is in reasonable agreement with literature.

\section{ACKNOWLEDGMENTS}

We gratefully acknowledge help from A. Manhard for the evaluation of the $\mathrm{H}_{2}$ Fulcher line intensities, W. Hohlenburger for the preparation of the cover electrodes and T. Dürbeck for technical assistance. This project has received funding from the EURATOM research and training programme 2014-2018.

\section{REFERENCES}

${ }^{1}$ S. Yoon, K. Tan, Rusli, and J. Ahn, "Modeling and analysis of hydrogen-methane plasma in electron cyclotron resonance chemical vapor deposition of diamond-like carbon," J. App. Phys. 91, 40-47 (2002).

${ }^{2}$ I. B. Denysenko, S. Xu, J. D. Long, R. P. Rutkevych, N. A. Azarenkov, and K. Ostrikov, "Inductively coupled $\mathrm{Ar} / \mathrm{CH}_{4} / \mathrm{H}_{2}$ plasmas for low-temperature deposition of ordered carbon nanostructures," J. Appl. Phys. 95, 2713 (2004).

${ }^{3}$ J. Zhou, I. T. Martin, R. Ayers, E. Adams, D. Liu, and E. R. Fisher, "Investigation of inductively coupled Ar and $\mathrm{CH}_{4} / \mathrm{Ar}$ plasmas and the effect of ion energy on DLC film properties," Plasma Sources Sci. Technol. 15, 714-726 (2006). 
${ }^{4}$ J. S. Kim, S. S. Jang, and W. J. Lee, Proc. Advanced Metallization and Interconnect Systems for ULSI Applications in 1995 Materials Research Society, Pittsburgh, 297 (1996). ${ }^{5}$ A. Weber, R. Nikulski, C.-P. Klages, M. Gross, R. Charatan, R. Opilan, and W. Brown, "Aspects of TiN and Ti deposition in an ECR plasma enhanced CVD process," Applied Surface Science 91, 314-320 (1995).

${ }^{6}$ M. S. Ameen, J. T. Hillman, J. Faguet, R. F. Foster, C. Arena, and F. Martin, Proceedings of the Advanced Metallization for ULSI Applications in 1994 Materials Research Society, Pittsburgh, 269 (1995).

${ }^{7}$ A. Efremov, N. Min, J. Jeong, Y. Kim, and K. Kwon, "Etching characteristics of $\mathrm{Pb}(\mathrm{Zr}, \mathrm{Ti}) \mathrm{O}_{3}, \mathrm{Pt}, \mathrm{SiO}_{2}$ and $\mathrm{Si}_{3} \mathrm{~N}_{4}$ in an inductively coupled $\mathrm{HBr} / \mathrm{Ar}$ plasma," Plasma Sources Sci. Technol. 19, 045020 (2010).

${ }^{8}$ R. A. Ditizio, G. Liu, S. J. Fonash, B.-C. Hseih, and D. W. Greve, "Short time electron cyclotron resonance hydrogenation of polycrystalline silicon thin-film transistor structures," Applied Physics Letters 56, 1140-1142 (1990).

${ }^{9}$ E. S. Cielaszyk, K. H. R. Kirmse, R. A. Stewart, and A. E. Wendt, "Mechanisms for polycrystalline silicon defect passivation by hydrogenation in an electron cyclotron resonance plasma," Appl. Phys. Lett. 67, 3099-3101 (1995).

${ }^{10}$ R. A. Gottscho, B. L. Preppernau, S. J. Pearton, A. B. Emerson, and K. P. Giapis, "Realtime monitoring of low temperature hydrogen plasma passivation of GaAs," J. Appl. Phys. 68, 440-445 (1990).

${ }^{11}$ J. D. Bernstein, S. Qing, C. Chan, and T.-J. King, "High dose-rate hydrogen passivation of polycrystalline silicon CMOS TFTs by plasma ion implantation," IEEE Trans. Electron Devices 43, 1876-1882 (1996).

${ }^{12}$ C.-F. Yeh, T.-J. Chen, C. Liu, J. Gudmundsson, and M. Lieberman, "Hydrogenation of polysilicon thin-film transistor in a planar inductive $\mathrm{H}_{2} / \mathrm{Ar}$ discharge," Electron Device Letters, IEEE 20, 223 -225 (1999).

${ }^{13}$ N. Fox-Lyon, G. S. Oehrlein, N. Ning, and D. B. Graves, "Hydrogenation and surface density changes in hydrocarbon films during erosion using $\mathrm{Ar} / \mathrm{H}_{2}$ plasmas," Journal of Applied Physics 110, 104314 (2011).

${ }^{14}$ S. Takashima, M. Hori, T. Goto, A. Kono, and K. Yoneda, "Absolute concentration and loss kinetics of hydrogen atom in methane and hydrogen plasmas," Journal of Applied Physics 90, 5497-5503 (2001).

${ }^{15}$ J. Jolly and J.-P. Booth, "Atomic hydrogen densities in capacitively coupled very highfrequency plasmas in $\mathrm{H}_{2}$ : Effect of excitation frequency," J. Appl. Phys. 97, 103305 (6pp) (2005).

${ }^{16}$ A. Rousseau, G. Cartry, and X. Duten, "Surface recombination of hydrogen atoms studied by a pulsed plasma excitation technique," Journal of Applied Physics 89, 2074-2078 (2001).

${ }^{17}$ M. Sode, T. Schwarz-Selinger, and W. Jacob, "Ion chemistry in $\mathrm{H}_{2}$-Ar low temperature plasmas," Journal of Applied Physics 114, 063302 (2013).

${ }^{18}$ A. D. Tserepi and T. A. Miller, "Two-photon absorption laser-induced fluorescence of H atoms: A probe for heterogeneous processes in hydrogen plasmas," Journal of Applied Physics 75, 7231-7236 (1994).

${ }^{19}$ P. Kae-Nune, J. Perrin, J. Jolly, and J. Guillon, "Surface recombination probabilities of 
$\mathrm{H}$ on stainless steel, a-Si:H and oxidized silicon determined by threshold ionization mass spectrometry in $\mathrm{H}_{2}$ RF discharges," Surface Science Letters 360, L495 (1996).

${ }^{20}$ Y. Abe, S. Kawashima, K. Takeda, M. Sekine, and M. Hori, "Dependence of surface-loss probability of hydrogen atom on pressures in very high frequency parallel-plate capacitively coupled plasma," Applied Physics Express 3, 106001 (2010).

${ }^{21}$ I. Méndez, F. J. Gordillo-Vazquez, V. J. Herrero, and I. Tanarro, "Atom and ion chemistry in low pressure hydrogen DC plasmas," The Journal of Physical Chemistry A 110, 60606066 (2006).

${ }^{22}$ M. Sode, T. Schwarz-Selinger, and W. Jacob, "Quantitative determination of massresolved ion densities in $\mathrm{H}_{2}$-Ar inductively coupled radio frequency plasmas," Journal of Applied Physics 113, 093304 (2013).

${ }^{23} \mathrm{~V}$. A. Kadetov, Diagnostics and modeling of an inductively coupled radio frequency discharge in hydrogen, PhD thesis, Ruhr Universität Bochum (2004).

${ }^{24} \mathrm{Macor}^{\circledR}$ is a registered trademark of Corning Inc.

${ }^{25}$ M. A. Lieberman and A. J. Lichtenberg, Principles of plasma discharges and materials processing (John Wiley and Sons, Inc., Hoboken, New Jersey, 2005).

${ }^{26}$ D. Wünderlich, S. Dietrich, and U. Fantz, "Applications of a collisional radiative model to atomic hydrogen for diagnostic purposes," Journal of Quantitative Spectroscopy and Radiative Transfer 110, 62 (2009).

${ }^{27}$ J. B. Boffard, R. O. Jung, C. C. Lin, and A. E. Wendt, "Optical emission measurements of electron energy distributions in low-pressure argon inductively coupled plasmas," Plasma Sources Sci. Technol. 19, 065001 (2010).

${ }^{28}$ J. E. Chilton, J. B. Boffard, R. Scott, and C. C. Lin, "Measurement of electron-impact excitation into the $3 \mathrm{p}^{5} 4 \mathrm{p}$ levels of argon using Fourier-transform spectroscopy," Phys. Rev. A 57, 267 (1998).

${ }^{29}$ T. Czerwiec, F. Greer, and D. B. Graves, "Nitrogen dissociation in a low pressure cylindrical ICP discharge studied by actinometry and mass spectrometry," J. Phys. D 38, 4278 (2005).

${ }^{30}$ A. Manhard, Spectroscopic Studies on Positive Ion Based Neutral Beam Injection Systems, Diploma thesis, Universität Augsburg (2008).

${ }^{31}$ U. Fantz, "Emission spectroscopy of molecular low pressure plasmas," Contrib. Plasma Phys. 44, 508-515 (2004).

${ }^{32}$ D. Wünderlich, (2013), private communication.

${ }^{33}$ H. R. Maurer, R. Basner, and H. Kersten, "Temperature of particulates in low-pressure rfplasmas in $\mathrm{Ar}, \mathrm{Ar} / \mathrm{H}_{2}$ and $\mathrm{Ar} / \mathrm{N}_{2}$ mixtures," Contributions to Plasma Physics 50, 954-961 (2010).

${ }^{34}$ S. Jacq, C. Cardinaud, L. L. Brizoual, and A. Granier, "H atom surface loss kinetics in pulsed inductively coupled plasmas," Plasma Sources Science and Technology 22, 055004 (2013).

${ }^{35} \mathrm{P}$. J. Chantry, "A simple formula for diffusion calculations involving wall reflection and low density," J. Appl. Phys. 62, 1141-1148 (1987).

${ }^{36}$ U. Cvelbar, M. Mozetič, I. Poberaj, D. Babič, and A. Ricard, "Characterization of hydrogen plasma with a fiber optics catalytic probe," Thin Solid Films 475, 12-16 (2005). 
${ }^{37}$ E. Tatarova, F. M. Dias, C. M. Ferreira, and N. Puac, "Spectroscopic determination of H, $\mathrm{He}$, and $\mathrm{H}_{2}$ temperatures in a large-scale microwave plasma source," J. Appl. Phys. 101, 063306 (2007).

${ }^{38}$ L. Tomasini, A. Rousseau, G. Gousset, and P. Leprince, "Spectroscopic temperature measurements in a $\mathrm{H}_{2}$ microwave discharge," Journal of Physics D: Applied Physics 29, 1006 (1996).

${ }^{39}$ G. Baravian, Y. Chouan, A. Ricard, and G. Sultan, "Doppler-broadened $\mathrm{H}_{\alpha}$ line shapes in a rf low-pressure $\mathrm{H}_{2}$ discharge," Journal of Applied Physics 61, 5249-5253 (1987).

${ }^{40}$ A. Rousseau, A. Teboul, and N. Sadeghi, "Time-resolved gas temperature measurements by laser absorption in a pulsed microwave hydrogen discharge," Plasma Sources Sci. Technol. 13, 166 (2004).

${ }^{41}$ T. Lamara, R. Hugon, and J. Bougdira, "Influence of gas temperature on the loss mechanisms of H-atoms in a pulsed microwave discharge identified by time-resolved LIF measurements," Plasma Sources Science and Technology 15, 526 (2006).

${ }^{42}$ A. M. Bruneteau, G. Hollos, R. Leroy, P. Berlemont, M. Bacal, and J. Bertagne, "Atomic temperature and density in multicusp $\mathrm{H}^{-}$volume sources," AIP Conference Proceedings 210, 504-515 (1990).

${ }^{43} \mathrm{M}$. Sode, Quantitative Beschreibung von Wasserstoff-Stickstoff-Argon-Mischplasmen, $\mathrm{PhD}$ thesis (in German), Universität Kiel (2013).

${ }^{44}$ M. Mozetič, M. Drobnič, and A. Zalar, "Recombination of neutral hydrogen atoms on AISI 304 stainless steel surface," Appl. Surf. Sci. 144-145, 399-403 (1999).

${ }^{45}$ Note that Abe et al. ${ }^{20}$ studied a plasma chamber where the upper electrode was made of aluminum but the lower electrode consisted of ceramic and Si. However, due to a low area of the Si wafer contributing to the total chamber surface and due to a considerably lower $\mathrm{H}$ loss probability on ceramic than on $\mathrm{Al}$ it is assumed that the dominant material causing the resulting $\mathrm{H}$ loss probability is $\mathrm{Al}$. 